\title{
An isolated colonic neurofibroma
}

\author{
Mohammad Bilala ${ }^{a}$, Farshaad Bilimoriaa ${ }^{b}$ Kofi Clarke ${ }^{a, c}$ \\ Allegheny Health Network, Pittsburgh, PA, USA
}

A 52-year-old male with a past medical history of hypertension presented with a two-week history of mild right lower quadrant abdominal pain. He denied chronic nonsteroid anti-inflammatory drug use, alcohol or tobacco abuse. No skin lesions or any areas of hypo/hyperpigmentation were noted. Abdominal exam revealed mild tenderness in the right lower quadrant. Laboratory data including serum sodium, potassium, creatinine and blood urea nitrogen were normal. Other normal tests included liver function tests, lipase and thyroid stimulating hormone levels. Urine analysis was negative. A colonoscopy was performed which showed three sessile $3 \mathrm{~mm}$ polyps in the splenic flexure and proximal descending colon (Fig. 1). Polypectomy was performed and histology examination showed a thickened lamina propria arranged in a fascicular-like pattern wrapped around dispersed benign colonic glands. Immunohistochemical staining with S-100 confirmed the diagnosis of colonic neurofibroma (Fig. 2).

Isolated neurofibroma of the gastrointestinal tract was first described in 1937, and remains a rare entity [1]. Since then, less than 20 such cases have been reported in the literature [2]. Clinical presentation ranges from incidental findings on routine colonoscopy to massive lower gastrointestinal bleeding [2,3]. In spite of an increase in reporting of isolated colonic neurofibromas (ICN) in the past decade, the etiology, pathogenesis, prognosis and treatment options for ICN remain unclear. In addition, there are no data on the need for follow-up colonoscopies and the appropriate intervals.

This and other cases highlight the importance of close follow up and detailed skin exams in these patient populations

Departments of a Internal Medicine (Mohammad Bilal, Kofi Clarke); bPathology (Farshaad Bilimoria); 'Gastroenterology, Hepatology and Nutrition (Kofi Clarke), Allegheny Health Network, Pittsburgh, PA, USA

Conflict of Interest: None

Correspondence to: Mohammad Bilal, 320 East North Avenue, Pittsburgh, PA 15212, USA, Tel.: +1 412660 3624, Fax: +1 412 359 4983, e-mail: mbilal@wpahs.org

Received 10 March 2016; accepted 10 March 2016 Published online 7 April 2016

DOI: http://dx.doi.org/10.20524/aog.2016.0029
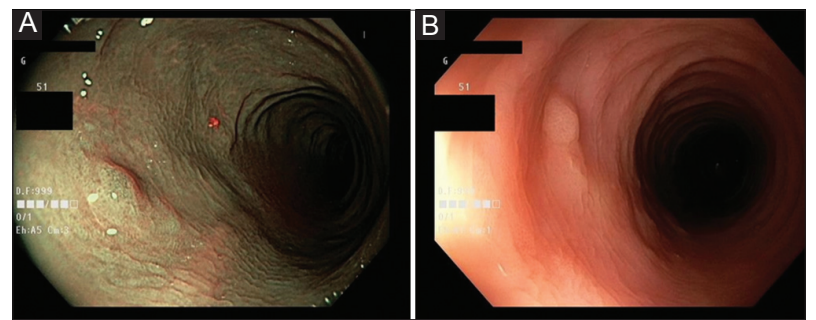

Figure 1 (A) Narrow band imaging showing sessile polyps in the proximal descending colon. (B) Colonoscopy with white light showing sessile polyps in the splenic flexure

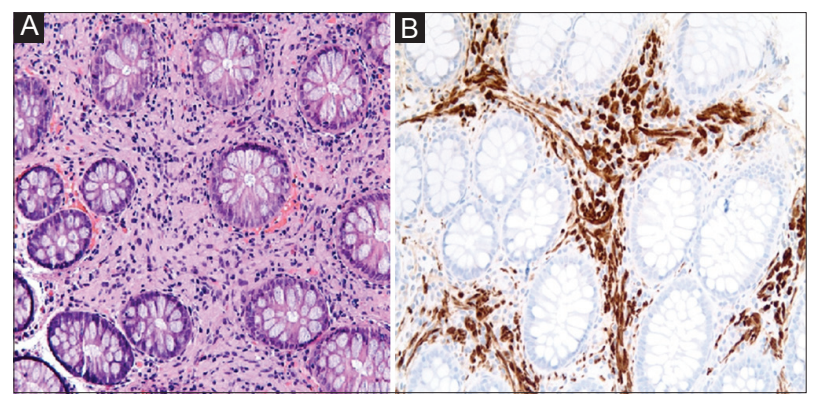

Figure 2 (A) Low magnification (20 x). H \& E stain. Specimen showing unremarkable large bowel glands separated by a thickened lamina propria. (B) Low magnification (20 x), S-100 stain. The cells of the lamina propria are positive for S-100 immunohistochemical stain, indicating a neurofibroma

to monitor for any signs of systemic neurofibromatosis. It also raises the need for a consensus guideline on follow-up care in patients with ICN including intervals for surveillance colonoscopies.

\section{References}

1. Keith AR. A case of neurofibromatosis of the rectal wall. Trans Am Proctol Soc 1937;38:68.

2. Chelimilla $H$, Chandrala $C K$, Niazi M, Kumbum K. Incidental finding of isolated colonic neurofibroma. Case Rep Gastroenterol 2013;7:369-375.

3. Abramson LP, Orkin BA, Schwartz AM. Isolated colonic neurofibroma manifested by massive lower gastrointestinal bleeding and intussusception. South Med J 1997;90:952-954. 